\title{
El pitching 2.0: conceptualización y desarrollo en proyectos de crowdfunding ${ }^{l}$
}

\author{
Marta MARTÍN-NúÑEZ \\ Universitat Jaume I de Castellón \\ mnunez@com.uji.es
}

\begin{abstract}
Resumen:
Los portales de crowdfunding invierten el proceso de producción tradicional al vender los proyectos audiovisuales directamente a los propios consumidores finales a través de vídeos e información escrita. En esta investigación nos proponemos conceptualizar el concepto de pitching 2.0 y su desarrollo en este contexto a través del análisis de proyectos audiovisuales del portal español Verkami.
\end{abstract}

Palabras clave: Crowdfunding; producción audiovisual; web 2.0; financiación; Internet; verkami

\section{Pitching 2.0: conceptualization and develpment in crowdfunding projects}

\begin{abstract}
Abtract:
Crowdfunding sites invert the traditional film production process selling audiovisual projects directly to end consumers through videos and written information. In this paper we aim to conceptualize the concept of pitching 2.0 and its development in this context through the analysis of audiovisual projects in the Spanish site Verkami.
\end{abstract}

Key words: Crowdfunding; film production; web 2.0; funding; Internet; verkami

Referencia normalizada:

Martín Nuñez, M. (2014): El pitching 2.0: conceptualización y desarrollo en proyectos de crowdfunding. Historia y Comunicación Social. Vol. 19. Núm. Especial Enero. Págs. 821-832.

Sumario: 1. Introducción y estado de la cuestión. 2. Metodología. 3. El pitching 2.0 en la presentación de proyectos audiovisuales. 3.1. Pitching 2.0: concepto. 3.2. Pitching 2.0: recomendaciones para su desarrollo. 3.2.1. Presentación escrita. 3.2.2. Vídeo de presentación. 3.2.3. Recompensas. 3.2.4. Creación de comunidad. 4. Conclusiones. 5. Referencias bibliográficas. 6. Referencias web.

\section{Introducción y estado de la cuestión}

Tradicionalmente, en el sector de la producción audiovisual, un productor financiaba un proyecto en base a su experiencia sobre lo que podría funcionar o no en el mercado audiovisual. Los guionistas se reunían con los productores para intentar vender sus creaciones y conseguir los medios necesarios para llevarlas a cabo en lo que se denominaba un pitching ${ }^{2}$. Hoy, las plataformas de crowdfunding o micro- 
mecenazgo surgen como una nueva tendencia que invierte este proceso. Portales de Internet como Lánzanos ó Verkami, permiten que usuarios anónimos puedan hacer microdonaciones para apoyar los proyectos que quieren ver realizados. Aunque encontramos proyectos de todo tipo en estos portales, abundan los de ámbito cultural y social que, en el contexto socioeconómico actual, no encuentran el apoyo institucional necesario para poder ser llevados a cabo por las vías tradicionales. En el ámbito de la producción audiovisual, este modelo permite que el público financie por adelantado, a modo de prepago, aquello que quiere ver, haciendo posible que proyectos profesionales y amateur encuentren una vía de financiación para ser producidos con más recursos que los aportados por los propios participantes del proyecto, los productores y las decrecientes subvenciones institucionales. Este cambio, por tanto implica que la producción se debe seguir vendiendo, pero ya no en el tradicional pitching con el productor, ni en la solicitud de una subvención, sino en una suerte de pitching 2.0: el proyecto que se utilizará en la página del portal de crowdfunding para solicitar financiación a los internautas.

Del crowdfunding como fenómeno nuevo o, por lo menos, renovado, poco se conoce aún sobre su uso y funcionamiento. Según los datos cedidos por Verkami para este estudio ${ }^{3}$ se han registrado 2049 proyectos, de los cuales 1833 han finalizado en éxito (casi un 90\%), recaudando 6.027.160 euros (sólo entre los proyectos finalizados con éxito) abonados por 154.644 mecenas desde diciembre de 2010, un periodo de tiempo que no llega a los 3 años. Estas cifras, sólo de uno de los portales de crowdfunding que operan en España, habla de la importancia del fenómeno como vía de financiación de proyectos culturales. En el sector de la producción de proyectos audiovisuales (categoría film: cortometrajes, largometrajes, documental, vídeo musical, series, webseries, animación, arte, eventos), se han registrado 442 proyectos, con una tasa de éxito de casi el 67\% (296 proyectos) y una recaudación de 1.603.604 euros aportados por 32.931 mecenas. En este contexto, la presentación del propio proyecto, es decir, el cómo se vende en una plataforma de crowdfunding, donde compite con muchos otros tantos proyectos por recursos económicos, se convierte en un aspecto primordial.

Por otra parte, no es labor fácil rastrear literatura científica sobre el concepto de crowdfunding, ya que la investigación en España sobre este tema es muy reciente y se remonta casi en su totalidad a los años 2012 y 2013, pese a que los primeros proyectos que utilizan esta estrategia de financiación se impulsan en 2009 (El cosmonauta [Nicolás Alcalá, 2013]). Además, la novedad del fenómeno y la variedad de disciplinas científicas desde las que se puede estudiar (economía, estructura de la comunicación, producción audiovisual, nuevas tecnologías de la comunicación, etc.) no contribuye a definir claramente los límites y el concepto. Nuestro punto de partida se sitúa en el monográfico Cine enredado. Nuevos contenidos y estrategias 2.0 publicado por L'Atalante. Revista de Estudios Cinematográficos dedicado a las nuevas tendencias del cine en Internet, en el que el fenómeno es abordado por especialistas.

Antoni Roig Telo, en Cine en abierto: formas y estrategias de producción basadas en la participación (2012), nos introduce al concepto de crowdfunding como una 
forma de crowdsourcing, que «hace referencia a la externalización de un proceso en manos de un amplio colectivo, de forma que contribuya a resolver un problema concreto a cambio de un reconocimiento afectivo más que económico» (Roig, 2012: 22). El crowdfunding, es una práctica de crowdsourcing enfocada específicamente a la financiación del proyecto. De esta forma, el «énfasis es en la financiación, de manera que se busca que el colectivo contribuya con pequeñas cantidades a cambio de un retorno, no tanto de la inversión, sino en forma de reconocimiento afectivo» (Roig, 2012: 23). Esta práctica no es nueva, pero la Web 2.0, los portales específicos en Internet y las redes sociales sí le han otorgado una nueva dimensión. Ya en 1913 Oscar Micheaux, un joven aforamericano hijo de esclavos liberados, financió la impresión de su primera novela, su autobiografía, con la preventa puerta a puerta de los ejemplares, todavía no publicados, con un capítulo de prueba. Más adelante, repitió la fórmula para llevar al cine su autobiografía, que Hollywood se había negado a producir (Barabas, 2012: 38). La trayectoria académica del profesor Roig Telo, experto en medios y participación social nos ha llevado a artículos donde explora el proceso de crowdfunding en profundidad, ligándolo a otras prácticas colaborativas. Curiosamente, su tesis, Cine colaborativo: prácticas culturales y formas de producción participativas (2008) prepara el terreno para dotar de un marco teórico este fenómeno pero sin nombrarlo como tal, ya que probablemente no estaba tan extendido bajo esa etiqueta en ese momento. Posteriormente, en artículos como ¡Esta película la hacemos entre todos! Crowdsourcing y crowdfunding como prácticas colaborativas en la producción audiovisual contemporánea (2012) o el ya citado publicado en L'Atalante sienta las bases del concepto definiéndolo e integrándolo en un marco más amplio de prácticas culturales colaborativas.

Otros trabajos de referencia genéricos son el libro Crowdfunding: La eclosión de la financiación colectiva, un cambio tecnológico, social y económico de Rivera Butzbach (2012) que describe el fenómeno de forma genérica y dedica un capítulo específico a cómo presentar el proyecto y otro dedicado al sector audiovisual, música, cine y video, que nos interesan especialmente. Y el libro Industrias audiovisuales: producción y consumo en el siglo XXI, que incluye un capítulo sobre Nuevas formas de financiación: Crowdfunding (Villalta et al., 2013) y se centra en conocer la viabilidad del proceso como nueva forma de producción.

Algunos de los trabajos están enfocados hacia la oportunidad financiera del fenómeno. Ruiz Gutiérrez, en Crowdfunding y Creative Commons: nuevos modelos de financiación y propiedad intelectual para la producción y distribución de proyectos audiovisuales (2010), aporta una idea interesante: «lo acertado sería hacer uso del modelo crowdfunding no tanto como una vía de financiación, sino como una herramienta para crear una comunidad activa e involucrada en la producción de la película» (2010: 44). Por otra parte, el artículo en el congreso Finançament alternatiu a l'era digital: crowdfunding i xarxes d'intercanvis. Fundació Catalunya Europa, Micro Financiación Colectiva (Crowd-funding): un nuevo instrumento económico para el crecimiento económico y el empleo. Un análisis de los efectos económicos de la Micro-financiación Colectiva con especial énfasis en el caso español (Ramos et al., 
2013) ofrece una visión panorámica que pretende contribuir a una mejor comprensión de este nuevo fenómeno económico, prestando especial atención a su efecto en la promoción de la actividad económica y el empleo, especialmente en España. Manejando cifras de 2012 adelantan que según estimaciones en torno a 3 billones de dólares se moverán en proyectos financiados a través de esta vía en 2013. En España, ejemplo de economía en recesión, esta forma de financiación ha atraído más de 400 millones de euros a través de 2.825 proyectos, que han creado 7.500 puestos de trabajo directos en 2012, es decir un empleo por cada 600 euros invertidos, lo que da cuenta de la magnitud económica del fenómeno.

Las investigaciones que se han realizado hasta el momento inciden sobre los aspectos más genéricos del crowdfunding como nueva forma de financiación de proyectos, pero no reflexionan sobre la presentación de los proyectos. Esta cuestión ha sido trabajada por la investigadora y dos trabajos previos sirven de marco para esta investigación sobre el pitching 2.0: (1) el artículo Crowdfunding en la producción audiovisual. Recomendaciones para la presentación de proyectos (Martín-Núñez, 2012) en el que se establecía el marco teórico para conocer en profundidad el concepto de crowdfunding, apoyado con datos sobre los proyectos facilitados por la plataforma Verkami cruzando la tasa de éxito y fracaso con las disciplinas, el presupuesto de los proyectos y la distribución geográfica y a través del análisis de casos, se establecían unas recomendaciones para la presentación de proyectos audiovisuales; y (2) Las presentaciones audiovisuales en los proyectos de crowdfunding (Martín-Núñez, 2013), donde se hacía un análisis de los vídeos de presentación de los proyectos.

\section{Metodología}

Como hemos indicado antes, este artículo supone la continuación de una línea de investigación que busca entender cómo funciona el sistema de crowdfunding en la producción audiovisual española. Muchos son los realizadores, profesionales y amateurs, que se han lanzado a esta aventura sin saber muy bien qué pedir, qué ofrecer, o cómo pedirlo, por lo que se estudiaron una serie de casos que llevaron a la elaboración de unas recomendaciones sobre la presentación de los proyectos (MartínNúñez, 2012). Dentro de estas recomendaciones, una de las partes analizadas más interesantes fueron precisamente los vídeos de presentación (Martín-Núñez, 2013). Lo que pretendemos ahora es y conceptualizar y desarrollar el pitching 2.0. a través del análisis de presentaciones de proyectos audiovisuales utilizados en los portales de crowdfunding, que se concreta en:

1. Definición del concepto de pitching 2.0

2. Identificación y desarrollo de las partes clave del pitching 2.0

Los análisis que nos han llevado a la definición del concepto pitching 2.0 han sido realizados sobre proyectos de Verkami, el portal español especializado en proyectos 
creativos que más ha crecido en los últimos dos años. Se han analizado 30 proyectos ya finalizados de la categoría film, independientemente de si han finalizado en éxito o fracaso. De estos proyectos, se ha analizado: tipo de proyecto, técnica, importe solicitado, importe conseguido, información sobre la sinopsis del proyecto, el equipo de producción, cómo invertir el dinero solicitado, la pieza audiovisual (tipo de pieza audiovisual, duración, información para el usuario, estándar de calidad), tipo de recompensas, número de apoyos recibidos, y potenciación de la comunidad en torno al proyecto (redirección a social media, actualizaciones en el blog, comentarios). Se ha diseñado un cuestionario para recoger la información relativa a estos ítems y después, al cruzar la información se han detectado las tendencias. A partir del procesamiento crítico de estos datos, se han desarrollado una serie de recomendaciones que han llevado a la definición y conceptualización del pitching 2.0.

\section{El pitching 2.0 en la presentación de proyectos audiovisuales}

\subsection{Pitching 2.0: Concepto}

El pitching 2.0 es la presentación de un proyecto, en este caso audiovisual, a pequeños inversores o micromecenas, con el fin de que inviertan económicamente para recaudar la financiación necesaria para llevarlo a cabo utilizando las plataformas de crowdfunding y haciendo uso de las herramientas de la web 2.0. para crear una comunidad activa en torno al proyecto.

\subsection{Pitching 2.0: Recomendaciones para su desarrollo}

El pitching 2.0 debe se desarrolla en cuatro partes clave que se deben desarrollar de forma adecuada:

\subsubsection{Presentación escrita}

La información escrita sobre el proyecto debe ser clara, concisa, sintética y con profundidad hipertextual. Las propias plataformas asesoran y dirigen el contenido que se publicará pidiendo cierta información, aunque al final, depende del usuario qué poner y cómo hacerlo. Tras el análisis realizado, la recomendación que realizamos es la siguiente:

- Qué: información sobre el proyecto. Puede incluir el dónde y el cuándo. En el caso de largometrajes y cortometrajes de ficción, además, se debe añadir una breve sinopsis sobre la historia.

- Quién: punto de gran importancia. Bien sea un proyecto amateur o profesional, es importante presentar a los creadores del proyecto y su experiencia previa. Muchas veces está relacionado con la justificación del proyecto. 
A veces, también, conviene presentar el personal artístico o técnico, si es de especial relevancia.

- Por qué: quizá sea el punto más importante. Argumentar bien por qué se quiere llevar a cabo el proyecto puede ser la clave para conseguir financiación.

- Para qué: especificar cuál es el objetivo de la campaña de crowdfunding, y en qué se empleará el dinero, desglosado si se cree necesario.

- Cómo: información adicional sobre cómo se desarrollará el proyecto. Puede ponerse en la página de presentación aunque recomendamos que se realice a través de las herramientas específicas como el blog o las actualizaciones, a medida que el proyecto vaya evolucionando.

- Más información: Es imprescindible que la redacción tenga profundidad hipertextual. Así, por ejemplo se pueden enlazar los nombres de los creadores a sus respectivas páginas web o su experiencia a proyectos ya realizados. También es útil proporcionar más referencias sobre el proyecto, como veremos en el apartado dedicado a la comunidad, si tiene página web o perfiles en redes sociales.

\subsubsection{Vídeo de presentación}

El vídeo que ubica como cabecera del proyecto en las plataformas de crowdfunding es una pieza de vital importancia para el proyecto porque funciona como la presentación del proyecto. Es, en muchas ocasiones, el primer (y único) contacto que tiene el posible mecenas con el trabajo que va a apoyar económicamente bien a través de la plataforma, o bien como vídeo compartido a través de las redes sociales. Por ello, la impresión que genere ese contenido audiovisual será determinante para conseguir apoyos. $\mathrm{Su}$ importancia aumenta en el campo de la producción audiovisual, objeto de análisis, ya que da una idea del nivel de calidad que puede tener el producto final.

Duración. Tratándose de piezas promocionales, la duración debe ser siempre la mínima posible para transmitir la idea principal y lograr los objetivos propuestos. Desde la valoración crítica de los diferentes casos, recomendamos duraciones de hasta un minuto para el teaser, hasta dos minutos para el tráiler y hasta tres minutos para reportajes elaborados con varias voces o presentaciones ficcionalizadas.

Información que se da al espectador. La información que se da al espectador varía según el objetivo que se haya marcado, aunque casi todos los casos destacan la propia historia que va a ser narrada y que, en resumidas cuentas, es aquello que mueve el proyecto hacia delante. No obstante, se suele incluir información adicional en torno a los siguientes ejes:

- Datos sobre el equipo de producción y artístico, especialmente si se cuentan con nombres relevantes

- Información sobre el estado de la producción, en casos en los que ésta ya está algo avanzada 
- Información del ambiente o contexto que justifica la necesidad de llevar a cabo el proyecto, aunque si la historia es potente, esta información debería ser secundaría, ya que no sería necesario justificarla más allá.

- Vínculos a otras vías de información, como cuentas en redes sociales, blog del proyecto o web, etc. con el objetivo de que el espectador que quiera saber más tenga las referencias para encontrar el proyecto en la red.

- Llamada a la acción del tipo: "Colabora con nosotros" para llevar al espectador a la acción, bien una colaboración económica directa o ayuda con la difusión del proyecto en sus redes.

Estándar de calidad. El estándar de calidad en el vídeo de presentación dará una idea al espectador (acertada o no) de la calidad de la producción final, por lo que lo más recomendable es ofrecer la misma calidad en el vídeo de presentación que la que se espera conseguir en la pieza una vez finalizada. De esta forma se evitará dar una mala primera impresión, si la presentación es mucho peor que el vídeo final y también levantar falsas expectativas si es mejor. Entre los parámetros que definen el estándar de calidad audiovisual podemos citar la calidad de imagen (dimensiones en píxeles si es digital o tamaño del negativo si es celuloide), la calidad de sonido (nitidez, volumen y timbre adecuados), la calidad de la grabación y la edición (iluminación y encuadre sugerentes, movimientos de cámara relevantes y justificados, puesta en secuencia para favorecer la comunicación del objetivo principal), y calidad de los efectos de postproducción y grafismo (un abuso de efectos predefinidos puede dar una apariencia amateur al vídeo).

Tipología de videos. Tomando los modelos de vídeos sobre los que hemos trabajando con anterioridad (Martín-Núñez, 2013) nos proponemos ahora desarrollarlos y ejemplificarlos.

1. Imagen estática. Este recurso incluye una o varias ilustraciones, fotografías o frames de la producción. Ayuda a dar a conocer el contexto y el ambiente que se va a generar en el proyecto final, pero es una opción muy limitada que no adelanta la textura de la producción final, lo que puede incomodar a los mecenas. Por otra parte, es una opción que permite mayor inmediatez por lo que puede ser una buena opción para casos en los que sea necesario montar el proyecto de crowdfunding en poco tiempo o no se tenga la disponibilidad de actores o equipo audiovisual necesario.

2. Cartel de la producción audiovisual. Generar un cartel de la película y colgarlo como cabecera del proyecto ayuda a difundir desde el minuto cero la identidad visual, aportando además la información sobre el equipo artístico y de producción principal. Esto puede actuar como reclamo, además de trasmitir el ambiente del proyecto de forma inmediata y eficaz. Los carteles de películas tienen unos rasgos muy definidos que permiten identificar de un simple vistazo los aspectos clave del proyecto. Se trata de otra opción válida pero limitada en un entorno multimedia. 
3. Imagen estática con banda sonora. Esta opción consiste en elaborar un vídeo estático tomando una imagen fija (una foto, un frame, una ilustración, el propio cartel de la producción, etc.) añadiéndole una pista de audio, de forma que suma a las características de los modelos anteriores las cualidades expresivas del sonido, bien sean de una canción o de un texto locutado. Realizado con estándares profesionales puede ser una forma interesante de añadir un plus a la opción previa. Recomendamos, en estos casos, que la duración no sea más de un minuto aproximadamente.

4. Boceto. Es una opción muy arriesgada que consiste en presentar el proyecto antes de estar finalizado. Por una parte, permite al mecenas observar el estado actual del proyecto, que puede ser positivo para ganar su confianza pero, por otra, se corre el riesgo de que se forme una idea equivocada de lo que pude ser la producción final. En casos muy concretos (series de animación terminadas a falta de sonorizar, por ejemplo) siempre y cuando el proyecto esté en condiciones de hacerse público, puede ser interesante.

5. Presentación ficcionalizada. El propio equipo de producción presenta el proyecto a través de una pequeña secuencia ficcionalizada. El tema más habitual es el de una reunión de trabajo donde una o dos personas hablan entre sí o miran directamente a cámara, en tono humorístico y distendido, aludiendo a sus características principales. No deben ser pretenciosos y no deben alargarse en exceso ya que su objetivo real es crear empatía con el mecenas, no hacerlo reír a carcajadas ni dar toda la información del proyecto. Es importante que este tipo de vídeos tengan la misma calidad audiovisual que el proyecto final, ya que de lo contrario puede parecer un video casero.

6. Teaser-trailer. El formato teaser-trailer es el más popular para los vídeos de presentación. Generan interés y curiosidad en el proyecto sin desvelar mucha información, por lo que es más fácil explotar la propuesta estética que la narrativa. Suele ser utilizado en producciones que están en fase de preproducción, en las que todavía no hay demasiado material audiovisual disponible, sólo el suficiente para introducir la historia, o el contexto o ambiente en el que se desarrolla. De nuevo, es importante que el teaser tenga la misma calidad audiovisual que el proyecto final y que esté vinculado al proyecto de crowdfunding, para su circulación en redes sociales.

7. Trailer. El tráiler es un formato muy similar al teaser, salvo que desvela mucha más información sobre la producción y es un poco más largo, por eso se utiliza especialmente en proyectos orientados a recaudar financiación para la postproducción y distribución donde la fase de rodaje ya está finalizada. El objetivo aquí es dar una visión donde se vea el material final de la producción al mismo tiempo que se genera interés y curiosidad por la propuesta narrativa y estética. Conviene vincularlo al final al proyecto de crowdfunding. Se corre el riesgo de no despertar la curiosidad de los mecenas, que ya ven el producto finalizado y 
no encuentran justificada la aportación económica, por lo que se recomienda, si se opta por esta fórmula, justificar el presupuesto en detalle.

8. Reportaje. Se trata de un vídeo que sigue todas las pautas de un reportaje clásico televisivo donde los creadores (o un presentador) presentan el proyecto, intercalados con alguna imagen del rodaje (si ya existe), del making of y donde pueden intervenir otras figuras de autoridad para respaldarlo. El estilo puede ir desde un vídeo muy informativo a un vídeo mucho más creativo, y tiene la ventaja de que está realizado pensando en el proyecto de crowdfunding, por lo que repasa los puntos clave de la campaña.

\subsubsection{Recompensas}

Las recompensas son una de las partes clave de del pitching 2.0. porque aunque es evidente que en muchos casos no será la razón principal por la que los mecenas van a participar económicamente en la producción, es la contraprestación a su apoyo y deben ser gestionadas con diligencia. En muchas de las plataformas recomiendan un número limitado de aportaciones, para no confundir a los mecenas, pero con variedad suficiente para acomodar todos los bolsillos. Algunas de las recompensas vinculadas a las producciones que se han ofrecido en los proyectos analizados:

- Modelo pre-pago: acceso a la película en formato digital antes de su estreno, película en formato digital, copia en DVD/BD, copia en DVD/BD edición especial, invitación para el estreno.

- Modelo gratificación: aparecer en los créditos de la película con diferente rango (según aportación), merchandising relacionado con la película, $\mathrm{CD}$ de la banda sonora, copia firmada del guion, copia firmada del plan de producción, fotografías fijas del rodaje, ilustraciones o prints del cartel, invitación para conocer al equipo, asistir al rodaje, aparecer como extra, invitación a la fiesta final de rodaje, o al estreno, etc.

\subsubsection{Creación de comunidad}

La creación de una comunidad activa en torno al proyecto que lo apoye y comparta en redes sociales y participe activamente es la parte que más importante del pitching 2.0. Puede estar compuesta por muchas personas conocidas y otras tantas desconocidas unidas por el interés común de sacar adelante la propuesta audiovisual. Por ello, es necesario atender y cuidar a la comunidad a través de las herramientas que proporcionan las plataformas para ello. En los proyectos analizados, estas herramientas estaban bastante o muy descuidadas y no ofrecían información útil y relevante para la comunidad. Por ello, se recomienda:

- Ser rápido y diligente en la respuesta a los comentarios y preguntas de la comunidad. Aunque no se sepa la respuesta, es necesario responder con amabilidad y cortesía a quienes están interesados en el proyecto. Incluso si escriben para felicitar a los creadores, agradecer el gesto nunca sobra. 
- Utilizar la herramienta de blog/actualizaciones que ofrecen las plataformas para hacer partícipes a los mecenas de la evolución del proyecto, que también es, en parte suyo. Es una forma muy fácil de agradecer el apoyo financiero pero que pocas veces se utiliza bien o de forma asidua. Una actualización semanal puede ser una buena frecuencia para mostrar los avances del proyecto o para obsequiar a los seguidores del proyecto (que no tienen por qué ser mecenas) con pequeños detalles, como fondos de escritorio para el ordenador y el móvil con motivos de la producción, o similares.

Por otra parte, para fomentar la difusión, la inmediatez y la consolidación de los vínculos con la comunidad, se recomienda complementar las herramientas que ofrecen las plataformas con otras más específicas en redes sociales (una página es Facebook y un perfil en Twitter) e incluso con la realización de una página web propia. Estas herramientas de social media, además de ayudar a difundir el proyecto, contribuirán a estrechar lazos con la comunidad. No es casualidad que todos los proyectos que presentaban una propuesta más profesional, dominaban mejor todas las herramientas sociales que aquéllos que presentaban propuestas más amateur.

\section{Conclusiones}

Esta investigación ha culminado con la conceptualización del término pitching 2.0 en proyectos de crowdfunding, que ha derivado del análisis de la situación del crowdfunding actual, gracias a datos cedidos por la plataforma Verkami y el análisis en profundidad de 30 campañas de la plataforma. Tras analizar una serie de ítems y cruzar las variables se han detectado casos y tendencias que han permitido elaborar una serie de recomendaciones para la elaboración del pitching 2.0 atendiendo a la presentación escrita, el vídeo de presentación, las recompensas y la creación de una comunidad activa. Este trabajo culmina una investigación acerca del crowdfunding en el que se han ido estudiando diferentes aspectos como el estado del crowdfunding España y recomendaciones para la presentación de los proyectos (Martín-Núñez, 2012), o el análisis de las piezas audiovisuales (Martín-Núñez, 2013) que ha dado como fruto la conceptualización y desarrollo del término pitching 2.0 en el entorno de crowdfunding. Como fenómeno en plena evolución y condicionado tecnológicamente, esta investigación no nos permite más que entender un poco mejor qué está sucediendo en el presente para desarrollar herramientas útiles para encarar el futuro de la producción audiovisual. 


\section{Bibliografía}

RIVERA BUTZBACH, E. (2012). CROWDFUNDING: La eclosión de la financiación colectiva, un cambio tecnológico, social y económico. Barcelona: Microtemas.

ROIG, A. (2012). "Cine en abierto: formas y estrategias de producción basadas en la participación”. En L'Atalante. Revista de Estudios Cinematográficos, 13, 20-27.

VILLALTA, Francisco Javier; ALMAZÁN MARTÍN, Javier; ANDRADES DELGADO, Víctor Manuel. (2013). "Nuevas formas de financiación: Crowdfunding”. En PÉREZ RUFI, José Patricio (Ed.), Industrias audiovisuales: producción y consumo en el siglo XXI. Málaga: Grupo de investigación Eumed.net.

5.1 Bibliografía Web

BARABAS, R. L. (2012). "Crowdfunding: Trends and Developments Impacting Entertainment Entrepreneurs". En NYSBA Entertainment, Arts and Sports Law Journal, 23(2), 38-40. Recuperado en: http://www.disantobowles.com/wp-content/uploads/2012/10/EASLJSummer121.pdf [28/09/2013].

BELLEFLAMME, P.; LAMBERT, T.; SCHWIENBACHER, A. (2012). "Crowdfunding: Tapping the Right Crowd". En: Social Science Research Network eLibrary, online, 1-34. Recuperado en: http://papers.ssrn.com/sol3/papers.cfm?abstract $\mathrm{id}=1578175[28 / 09 / 2013]$.

DÍAZ, A. (2012). "Principales plataformas de crowdfunding nacionales e internacionales". En: Universocrowdfunding.com. Recuperado en: http://www.universocrowdfunding.com/principales-plataformas-crowdfunding/ [28/09/2013].

HERNANZ, M. (2012, octubre 9). “¿Funciona el crowdfunding cultural en España?” En: RTVE.es. Recuperado de: http://www.rtve.es/noticias/20121009/crowdfunding-cultural-espana/568606.shtml [28/09/2013].

MARTÍN-NÚÑEZ, M. (2012). "Crowdfunding en la producción audiovisual. Recomendaciones para la presentación de proyectos". En R. ZALLO ELGUEZABAL \& A. CASERO-RIPOLLÉS (Eds.), Comunicación y regeneración democrática. Actas del IV Congreso Nacional ULEPICC España (pp. 782-802). Presentada en el IV Congreso Nacional ULEPICC España, Universitat Jaume I, Castellón. Recuperado en: http://www.ulepicc.es/web/documentos/ACTAS_ULEPICC_2013.pdf [28/09/2013].

(2013). "Las presentaciones audiovisuales en los proyectos de crowdfunding". GONZÁLVEZ VALLÉS, J.E. (Coord.): Innovación: Redes sociales y los 2.0 y 3.0. Madrid: Vision Libros [en prensa].

RAMOS, Javier; GONZÁLEZ, Bruno; LLORCA, Ricardo; MAYOR, Luis; PORCEL, Rafa. (2013). "Micro Financiación Colectiva (Crowd-funding): un nuevo instrumento económico para el crecimiento económico y el empleo. Un análisis de los efectos económicos de la Micro-financiación Colectiva con especial énfasis en el caso español'. Presentada en Finançament alternatiu a l'era digital: crowdfunding i xarxes d'intercanvis. Fundació Catalunya Europa, Barcelona. Recuperado en: http://www.catalunyaeuropa.net/img/pdf/Paper_CF_Ateneu.pdf [28/09/2013]. 
ROIG TELO, A.; SÁNCHEZ-NAVARRO, J.; LEIBOVITZ, T. (2011). “EEsta película la hacemos entre todos! Crowdsourcing y crowdfunding como prácticas colaborativas en la producción audiovisual contemporánea". Revista ICONO14. Revista científica de Comunicación y Tecnologías emergentes, 10(1), 25-40. doi: http://dx.doi.org/10.7195/ri14.v10i1.113 [28/09/2013].

RUIZ GUTIÉRREZ, José. (2010). "Crowdfunding y Creative Commons: nuevos modelos de financiación y propiedad intelectual para la producción y distribución de proyectos audiovisuales." Cuadernos de Comunicación, 4. Recuperado en: http://cdc.escogranada.com/2010/12/crowdfunding-y-creative-commons-nuevos-modelos-de-financiacion-y-propiedad-intelectual-para-la-produccion-y-distribucion-de-proyectos-audiovisuales/ [28/09/2013].

VERKAMI (2012). Datos de crowdfunding actualizados a 2 de octubre de 2012. Recuperado en: https://docs.google.com/spreadsheet/pub?key=0AkO9AbAdjbTqdHRTVlpvZlJjek1TQ3B2SmZjRTJWbnc\&output=html [28/09/2013].

\section{Notas}

1 El presente estudio ha sido financiado con la ayuda del Proyecto de Investigación de la convocatoria Universitat Jaume I-Bancaja, con el título Análisis de los flujos de transferencia de conocimiento entre los sistemas educativos superiores y la industria del videojuego, código 11I301.01/1, para el periodo 2012-14, bajo la dirección del Dr. Javier Marzal Felici.

2 En inglés, una de las acepciones de pitch es la formulación para persuadir a alguien para que compre o acepte algo (Oxford Dictionaries)

3 Nos gustaría agradecer la amabilidad del portal Verkami y de sus empleados por ceder los datos sobre los proyectos de su plataforma hasta el 30 de septiembre de 2013 de forma desinteresada para esta investigación.

\section{La autora}

Marta Martín Núñez es doctora europea en Comunicación por la Universitat Jaume I de Castellón, donde ejerce como profesora e investigadora. Sus líneas de investigación se centran en la teoría de la imagen y las nuevas tecnologías digitales de la comunicación, trabajando cuestiones sobre fotografía, cine y videojuegos. Es miembro del consejo de redacción de L'Atalante. Revistas de Estudios Cinematográficos, adjunta a la redacción de Archivos de la Filmoteca, miembro del Comité editorial de las Guías para ver y analizar cine (Nau Llibres), forma parte de la secretaría técnica de adComunica. Revista Científica de Estrategias, Tendencias e Innovación en Comunicación y es miembro de la Asociación Española para la Investigación en Comunicación (AE-IC). Además, posee experiencia profesional en el campo de la publicidad y de la producción audiovisual. 